\title{
Numerical Analysis of Flow Field in Carbon Tank based on Fluent
}

\author{
Lu Shao-Yun, Wang Yin-Shan, Yang Shuai-Jun \\ School of Automobile and Transportation, \\ Tianjin University of Technology and Education, \\ Tianjin 300222, China
}

\begin{abstract}
In order to solve the problem of pollutant emission control of light vehicles in China, combined with the specific requirements of the actual project, Fluent software was used to conduct modeling and calculation of activated carbon canister of a certain vehicle, and the porous media model was introduced to analyze the distribution law of flow field inside the carbon tank, and the internal pressure and velocity distribution of the carbon tank under different working conditions were obtained.
\end{abstract}

Key words: Carbon tank, Fluent, porous media

With the continuous development and expansion of China's economy, the automobile industry and output occupy a large proportion in the national economy, which also causes serious environmental pollution, irreversible damage to the environment, and seriously endangers human health. In order to protect the environment and human health, it is necessary to control the evaporation emission of automobile pollution. The core of its evaporation emission control system is the carbon tank. The adsorption capacity of the carbon tank is applied to adsorb fuel vapor ${ }^{[1]}$. The porous media theory is used to simulate activated carbon particles, and Fluent software is used to simulate the internal flow field of the activated carbon tank used in vehicles.

Many scholars at home and abroad use numerical simulation to simulate the refueling process.

Hassanvand et al. used CFD numerical simulation method to simulate the evaporation of oil and gas during the splashing process of the oil tank. By using the fluid volume method and combining with the mass transfer model, they conducted experiments, and discussed the influence of loading speed, temperature and initial oil and gas mass fraction on gasoline volatilization loss ${ }^{[2]}$.

Miao Lei of Tianjin University studied the rule of evaporation emission of fuel and the influence of fuel temperature, air velocity and oil film thickness on evaporation emission. The process of fuel evaporation was numerically simulated by Fluent, and the flow and control equation of fuel evaporation model were described in detail. The reasons for the difference between simulation results and actual results are compared and analyzed ${ }^{[3]}$.

Zhang yun of Jiangsu University studied the distribution law of the flow field in the oil tank during the refueling process with the application of numerical simulation method, built a mathematical model with the userdefined Function UDF(user-define Function), and observed and studied the flow law during the refueling process with the simulation cloud map ${ }^{[4]}$. The results show that there are some errors between the experimental results and the simulation results, but they are consistent with each other.

\section{DEFINITION OF POROUS MEDIA AND RELATED PARAMETERS}

The simulation of flow field in the carbon tank is to simulate the adsorption and desorption process of the carbon tank. Due to the complex structure of the pores inside the carbon powder, the internal structure is in line with the basic characteristics of the porous medium structure. Therefore, porous media model is introduced to simulate the internal structure of activated carbon ${ }^{[5]}$.

\subsection{Definition of porous media}

A porous medium is a solid material that penetrates a large number of pores filled with a gas, liquid, or heterogeneous mixture. It's simply a solid material with lots of pores. It is characterized by having a solid skeleton, and the pores contain at least one non-solid substance, which can be liquid or gaseous ${ }^{[6]}$.

\subsection{Porous media related parameters}

(1) porosity

The pores in porous media are divided into effective pores and dead-end pores. The fluid in effective pores can flow freely, while the fluid in dead end pores is relatively stagnant [7]. The ratio of the total volume $V_{p}$ of the tiny voids in the porous media to the external volume $V_{t}$ of the porous media is called the porosity of the porous media, which is an important parameter affecting the fluid transport performance in the porous media.

$$
\varepsilon=\frac{V_{p}}{V_{t}}
$$

\section{(2) specific surface area}

Specific surface area is an important parameter affecting the performance of porous media, and it represents the internal surface area of pores per unit volume ${ }^{[8]}$.

$$
S=\frac{A}{V}
$$


Formula: $\mathrm{S}$ is the specific surface area;

A is the internal surface area of the pore;

$\mathrm{V}$ is the volume of porous medium.

(3) the porous medium resistance coefficient calculation formula

Specific parameters of viscosity resistance coefficient $1 / \alpha$ and inertia resistance coefficient $C_{2}$ need to be set in Fluent software. The size of resistance coefficient in porous media is calculated by the porosity and particle diameter of porous media.

Coefficient of viscous resistance:

$$
\frac{1}{\alpha}=\frac{150}{D_{p}^{2}} \frac{(1-\varepsilon)^{2}}{\varepsilon^{3}}
$$

Coefficient of inertia resistance:

$$
C_{2}=\frac{3.5}{D_{p}} \frac{(1-\varepsilon)}{\varepsilon^{3}}
$$

In the formula, $\varepsilon$ is the porosity; $D_{p}$ is the particle diameter.

\section{MODEL DESIGN AND MODEL MESHING}

\section{1 model design}

Figure 2 model is built based on the actual size of activated carbon canister figure 1 and UG $3 \mathrm{~d}$ modeling software. This carbon canister has three ports for adsorption and desorption experiments: desorption port, adsorption port and atmosphere port. The diameter of the adsorption port is $6 \mathrm{~mm}$, and the cross-sectional area is $1.13 \times 10^{-4} \mathrm{~m}^{2}$. The diameter of the desorption port is $8 \mathrm{~mm}$, the cross-sectional area is $5.02 \times 10^{-5}$ $\mathrm{m}^{2}$, the diameter of the air outlet is $6 \mathrm{~mm}$, and the crosssectional area is $1.13 \times 10^{-4} \mathrm{~m}^{2}$ 。
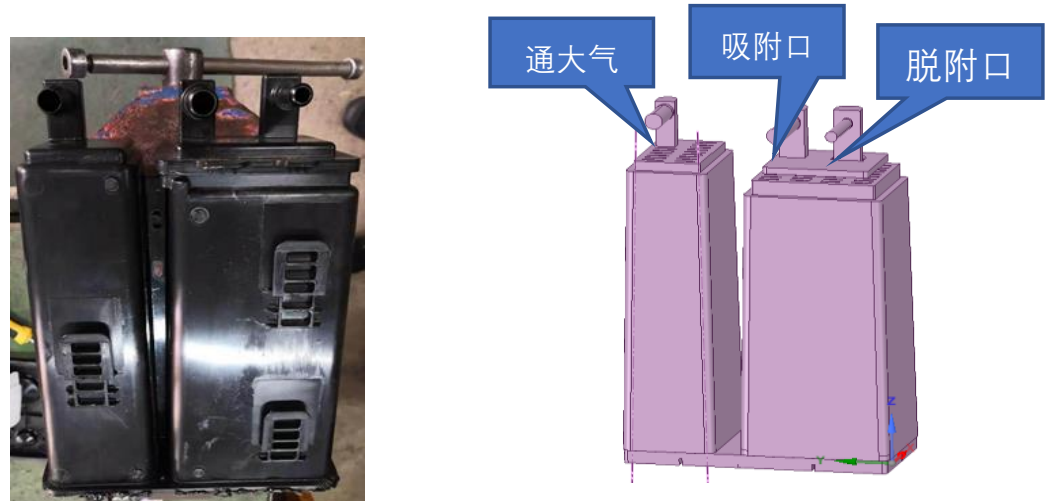

\section{2 model mesh generation}

The quality of grid generation directly affects the efficiency, accuracy and feasibility of calculation results. Ansys software is used to model and mesh the carbon canister. After the carbon tank was completely meshed, the number of meshes was about 3.735 million. In order to conduct material exchange in adjacent areas, interfaces were set on different contact surfaces, and the meshed files were imported into Fluent for analysis. Grid generation of flow field in carbon tank is shown in the figure.
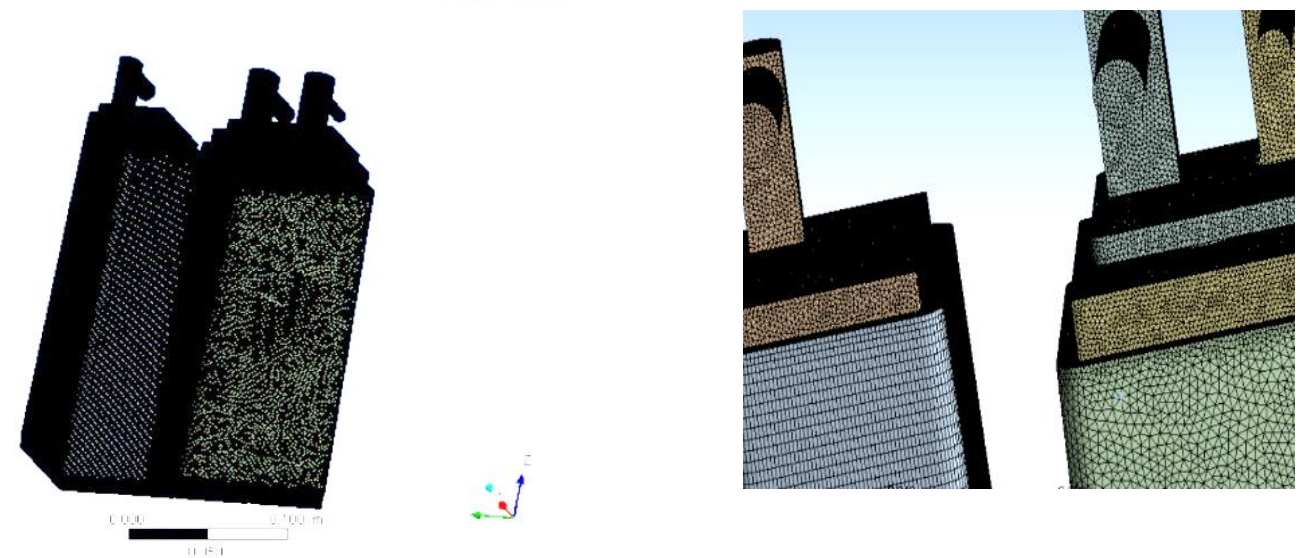


\section{SIMULATION ANALYSIS OF ADSORPTION PROCESS}

\section{1 definition of solver}

To simulate the adsorption process, assuming that activated carbon tank flow for steady state flow, set up the entrance velocity of $1500 \mathrm{l} / \mathrm{h}$, solver type selection based on the method of pressure solver (pressure - -based), time steadystate type selection, choose the absolute speed velocity equation and physical model simulation process selection standard $\mathrm{k}$ - epsilon turbulence model, algorithm USES the SIMPLE calculation method, discrete format using first-order windward difference.

\section{2 setting boundary conditions}

Boundary conditions are divided into several categories, including: inlet boundary conditions (velocity inlet, pressure inlet, etc.), outlet boundary conditions (pressure outlet), wall conditions, etc.

When simulating the adsorption process of activated carbon canister, the desorption port of activated carbon canister is closed, and the desorption port is set as the wall boundary. The adsorption port is set as the inlet and the atmospheric port as the outlet. The inlet boundary condition is velocity inlet boundary, the outlet boundary condition is pressure outlet boundary, and the fluid material is gasoline steam.

The area of powdered carbon used in the numerical simulation experiment was set as Porous medium type, the porosity of the used carbon powder $\varepsilon=0.32$, the particle diameter of the powdered carbon was $D_{p}=0.002 \mathrm{~m}$, and the Porous media zone was activated in FLUENT software, and the coefficient of viscous resistance and inertial resistance in different directions could be input. The viscosity resistance coefficient of the powder was obtained by substituting various parameters of the powder into the formula: $1 / \alpha=5.29 \times 10^{8}$, inertia resistance coefficient $C_{2}=36300$.

$3.31500 \mathrm{l} / \mathrm{h}$ adsorption process, the simulation results and analysis

The diagram shows the adsorption process of the carbon tank at a rate of $1500 \mathrm{~L} / \mathrm{h}$. After post-processing software, the pressure distribution diagram and velocity flow diagram of the flow field in the carbon tank are obtained.
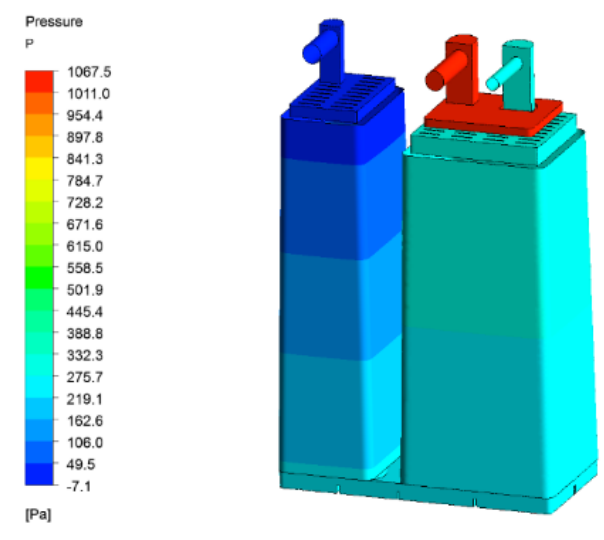

It can be seen from the three-dimensional pressure distribution diagram of the carbon tank that there is a significant pressure distribution at the atmospheric opening and adsorption opening, and the pressure range of the atmospheric opening is about $-7.1--49.5 p$, and the pressure at the adsorption opening is the largest, about 1000P. The pressure distribution in the flow field area of the carbon tank is uniform and has obvious pressure gradient.
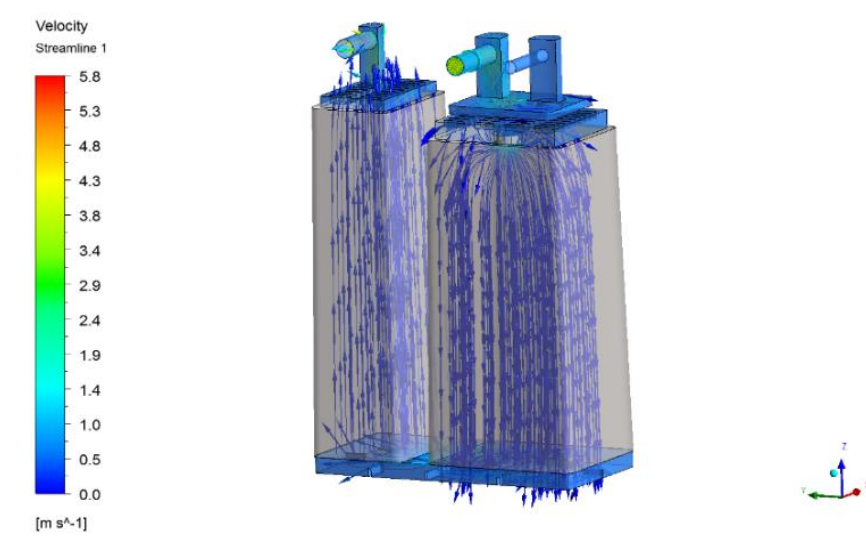
It can be seen from the flow chart that the flow field area in the whole carbon tank is mainly concentrated in the adsorption port and atmospheric port area with high velocity, and the flow velocity in other positions of the flow field is not high, and the flow velocity in the carbon powder area is below $0.5 \mathrm{~m} / \mathrm{s}$.

\section{SIMULATION ANALYSIS OF DESORPTION PROCESS}

\section{1 define solver}

The desorption process was simulated. The flow in the activated carbon tank was assumed to be steady state flow, and the inlet velocity was set at $2000 \mathrm{~L} / \mathrm{h}$. The solver type was selected in accordance with the adsorption process as pressure-based solver, and the standard k-erasure turbulence model was selected.

\section{2 setting boundary conditions}

When the desorption process is simulated, the adsorption port is considered to be closed and set as the wall boundary. Set the air port as the entrance and the desorption port as the exit. The inlet boundary is velocity inlet boundary and the outlet boundary is pressure outlet boundary. Fluid materials are selected for fuel vapor.

For the setting of the toner area, the porous medium type is adopted. The viscosity resistance coefficient of the powder was obtained by substituting various parameters of the powder into the formula: $1 / \alpha=5.29 \times 10^{8}$, inertia resistance coefficient $C_{2}=36300$.

$4.32000 \mathrm{l} / \mathrm{h}$ stripping process simulation results and analysis

The diagram shows the desorption process of the carbon tank at a rate of $2000 \mathrm{~L} / \mathrm{h}$. After post-processing software, the pressure distribution diagram and velocity flow diagram of the flow field in the carbon tank are obtained.

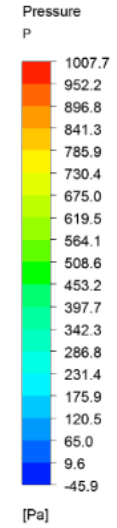

It can be seen from the three-dimensional pressure distribution diagram that the atmospheric opening and the desorption opening have obvious pressure distribution, and the pressure at the atmospheric opening is the largest, about $1000 \mathrm{P}$,
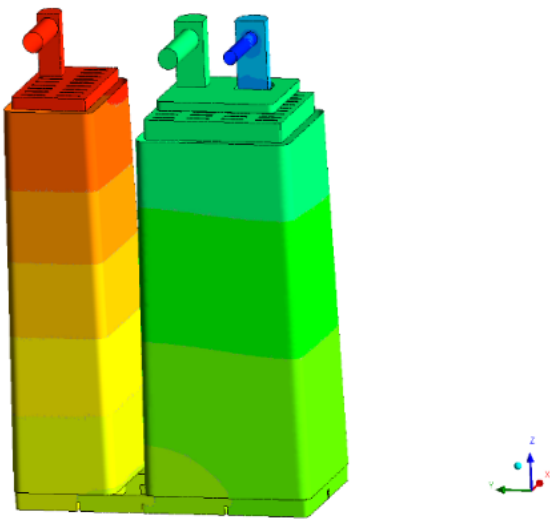
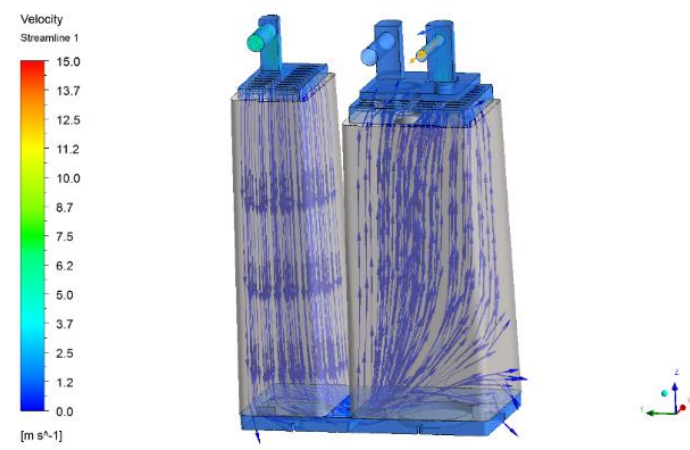

It can be seen from the flow chart that the flow field area in the whole carbon tank is mainly concentrated in the area of desorption port and atmospheric port with high velocity, while the flow velocity in other positions of the flow field is not high, and the flow velocity in the carbon powder area is below $1.2 \mathrm{~m} / \mathrm{s}$.

\section{SUBTOTAL}

Based on edge ford SUV imports gasoline vehicle and the pressure range of the desorption opening is about -45.9 -- 65.0p. The pressure distribution in the flow field area of the carbon tank is relatively uniform. activated carbon tank as the research object, using threedimensional modeling software is used for numerical simulation of carbon tank shell model is established, the use of Fluent software for related models of regional division, the setting of boundary conditions and mesh, into the porous medium model, got the canister adsorption stripping process of stress and velocity distribution contours. 


\section{REFERENCE}

[1] Wei haiyan, He ren. Research on the control technology of evaporative pollutants in gasoline vehicle on agricultural mechanization $[\mathrm{J}]$. Research on agricultural mechanization, 2008(1):196-199.

[2] A.Hassanvand, S.H.Hashemabadi, M.Bayat. Evaluation of gasoline evaporation during the tank splash loading by CFD techniques[J]. International Communications in Heat and Mass Transfer,2010,37(7).

[3] Miao lei. Research on vehicle fuel evaporation emission characteristics [D]. Tianjin university, 2010.

[4] Zhang yun. CFD analysis of fuel evaporation emission during refueling [D]. Jiangsu university,2017.

[5] Tong liang. Simulation and optimization of transport and adsorption in porous structure of carbon-based hydrogen storage materials [D]. Wuhan university of technology,2008.

[6] Chen yusun. New development of seepage theory -- comments on the Chinese translation of fluid dynamics in porous media [J]. Engineering investigation,1983(03):20-21.

[7] Yan meijuan. Some flow characteristics of fluids in porous media [D]. Huazhong university of science and technology,2008.

[8] Zhu jie. Phase change heat and mass transfer in porous media [D]. Dalian university of technology,2006. 